$10-21-2015$

\title{
Analysis of Speech-Language Pathology Graduate Students' Experience with Thickened Liquids
}

\author{
Mary Gorham-Rowan \\ Valdosta State University, mmgorhamrowan@valdosta.edu \\ Jade Coston \\ Valdosta State University
}

Follow this and additional works at: https://nsuworks.nova.edu/ijahsp

Part of the Speech and Hearing Science Commons, and the Speech Pathology and Audiology Commons

\section{Recommended Citation}

Gorham-Rowan M, Coston J. Analysis of Speech-Language Pathology Graduate Students' Experience with Thickened Liquids. The Internet Journal of Allied Health Sciences and Practice. 2015 Oct 21;13(4), Article 12.

This Manuscript is brought to you for free and open access by the College of Health Care Sciences at NSUWorks. It has been accepted for inclusion in Internet Journal of Allied Health Sciences and Practice by an authorized editor of NSUWorks. For more information, please contact nsuworks@nova.edu. 


\title{
Analysis of Speech-Language Pathology Graduate Students' Experience with Thickened Liquids
}

\begin{abstract}
Purpose: Thickened liquids are frequently recommended for patients with dysphagia to minimize aspiration. When discussing the use of thickened liquids with graduate students in Communication Sciences \& Disorders (CSD), it is beneficial to discuss the potential effect(s) of this dietary recommendation. Graduate level preparation involving first-hand experience with thickened liquids may help future speech-language pathologists (SLPs) develop a more complete understanding of the effects of diet modification. The purpose of the current study was to examine the reactions of graduate students in CSD to a brief period of thickened liquid use as an experiential learning activity. Methods: Sixtyeight graduate students enrolled in an accredited CSD program completed the study. As part of a class assignment, students were instructed to consume only thickened liquids for 24 hours. No further restrictions on food intake were made. Each student wrote a brief summary detailing his/her experience at the conclusion of the thickened liquid intake session. The narratives were examined for key terms that seemed to define the participants' experiences. Results: Experiential terms were divided into physical symptoms, emotional responses, and behavioral changes. Common symptoms included persistent thirst, dryness, fatigue, perception of the experience as being unpleasant, irritability, and difficulty concentrating. Conclusion: Many of the symptoms were associated with dehydration secondary to reduced fluid intake, per the students' reports. Specific symptoms included craving water, fatigue, and headache. All participants reported a greater understanding of thickened liquid use upon completion.
\end{abstract}

\section{Author Bio(s)}

Mary Gorham-Rowan, PhD, CCC-SLP, is a Professor of Communication Sciences and Disorders at Valdosta State University in Valdosta, Georgia.

Jade H. Coston, PhD, CCC-CSLP, is an Assistant Professor in Communication Sciences and Disorders at Valdosta State University. 


\title{
TIAHSP \\ The Internet Joưtnal of Allied Health Sciences and Practice
}

Dedicated to allied health professional practice and education

Vol. 13 No. 4 ISSN 1540-580X

\section{Analysis of Speech-Language Pathology Graduate Students' Experience with Thickened Liquids}

\author{
Mary Gorham-Rowan, $\mathrm{PhD}^{1}$ \\ Jade Coston, $\mathrm{PhD}^{2}$
}

1. Valdosta State University, Professor, Dept. of Communication Sciences and Disorders, Valdosta, GA

2. Valdosta State University, Associate Professor, Dept. of Communication Sciences and Disorders, Valdosta, GA

United States

\begin{abstract}
Purpose: Thickened liquids are frequently recommended for patients with dysphagia to minimize aspiration. When discussing the use of thickened liquids with graduate students in Communication Sciences \& Disorders (CSD), it is beneficial to discuss the potential effect(s) of this dietary recommendation. Graduate level preparation involving first-hand experience with thickened liquids may help future speech-language pathologists (SLPs) develop a more complete understanding of the effects of diet modification. The purpose of the current study was to examine the reactions of graduate students in CSD to a brief period of thickened liquid use as an experiential learning activity. Methods: Sixty-eight graduate students enrolled in an accredited CSD program completed the study. As part of a class assignment, students were instructed to consume only thickened liquids for 24 hours. No further restrictions on food intake were made. Each student wrote a brief summary detailing his/her experience at the conclusion of the thickened liquid intake session. The narratives were examined for key terms that seemed to define the participants' experiences. Results: Experiential terms were divided into physical symptoms, emotional responses, and behavioral changes. Common symptoms included persistent thirst, dryness, fatigue, perception of the experience as being unpleasant, irritability, and difficulty concentrating. Conclusion: Many of the symptoms were associated with dehydration secondary to reduced fluid intake, per the students' reports. Specific symptoms included craving water, fatigue, and headache. All participants reported a greater understanding of thickened liquid use upon completion.
\end{abstract}

\section{INTRODUCTION}

The use of thickened liquids as a means of providing hydration is a common recommendation for liquids. ${ }^{1}$ The underlying premise is that the use of thickened liquids allows for greater control of the liquid bolus, thereby increasing the time needed to trigger the pharyngeal swallow. Enhanced control of the liquid bolus minimizes the possibility that liquid will spill into the airway prematurely and thus improve airway protection. ${ }^{1,2}$ As noted in a review by Sura et al, there has been little evidence to support the effectiveness of thickened liquids. ${ }^{3}$ Although honey thick or "ultrathick" liquids may reduce aspiration for some patients, the overall effectiveness may be altered by timing or manner of presentation. Goulding and Bakheit demonstrated that thickened liquids eliminated aspiration in only one out of 10 patients. ${ }^{4}$ Furthermore, the most commonly recommended consistency is that of nectar thick, which is not consistent with the reports of greater effectiveness with thicker consistencies. ${ }^{1}$

The inherent nature of thickened liquids often contributes to a reduced fluid intake in patients with dysphagia. Patients frequently report that they do not like thickened liquids, thus reducing the level of compliance and reduced overall fluid intake. ${ }^{1} \mathrm{~A}$ number of studies have documented that hospitalized patients do not meet the minimum daily fluid requirement when placed on thickened liquids.5-7 McGrail and Kelchner noted several factors affecting fluid intake, including beverage preference and inaccurate preparation of thickened liquids. ${ }^{5}$ Health care providers are rarely trained and/or do not follow appropriate protocols in preparing 
thickened liquids, thus frequently serving patients liquids that are too thick or too thin.8,9 Poor preparation of thickened liquids further decreases the likelihood that patients will consume the liquids, thereby decreasing overall fluid intake.

As a result of the reduction in fluid intake, patients with dysphagia are at risk for dehydration. ${ }^{7}$ In the rehabilitation setting, dehydration has a number of consequences for patients. Mild dehydration has been shown to contribute to reduced physical, visuomotor, and psychomotor performance, as well as fatigue and reduced alertness. 10-13 Grandjean and Grandjean note that a loss of only $2 \%$ body weight can affect these functions. ${ }^{11}$ Declines in cognitive function have been reported in both adolescents and adults, including older adults. ${ }^{14-16}$ Given that many patients in rehabilitation settings who are prescribed thickened liquids may have cognitive and/or physical deficits associated with traumatic brain injury or stroke, the use of thickened liquids may further compromise their abilities in these areas and prolong the time needed to regain maximum function.

Despite the negative reactions of patients to thickened liquids and the lack of data to support their effectiveness, speechlanguage pathologists (SLPs) frequently employ them as a treatment strategy. One possible explanation for this discrepancy may be inadequate comprehension of the advantages and disadvantages of this type of diet modification. A superficial comprehension of a topic may be gained through traditional learning avenues, e,g., reading and classroom discussion. Comprehension is often enhanced, however, when learners experience a specific situation. Active learning is an evidence-based teaching strategy that encourages student participation in gathering information, reflective thinking, and problem solving. ${ }^{17}$ Graduate level preparation involving first-hand experience with thickened liquids may help future SLPs develop a more complete understanding of the effects of diet modification. The purpose of the current study, therefore, was to retrospectively examine the reactions of graduate student SLPs to a brief period of thickened liquid use as part of a scheduled class assignment.

\section{METHODS}

Participants. Sixty-eight young adults, 22 to 35 years of age, completed the assignment as part of a graduate level class in swallowing and swallowing disorders. Three of the students were male. All participants were in good health per self-report. Any individuals with a history of medical disorders that could be negatively impacted by changes in food/liquid intake were excluded from the assignment, e.g., individuals with urinary or bladder dysfunction, bowel disturbances, cardiac disorders, high blood pressure, diabetes, or hypoglycemia. These students completed an alternative assignment examining written research regarding the use of thickened liquids.

All of the students were familiar with the concept of thickened liquids as a management strategy, either through class discussions or direct patient observations. Most of them reported that they had learned about thickened liquids during their undergraduate studies. Their exposure to thickened liquids frequently involved either sampling a thickened beverage or observing the use of thickened liquids in a skilled nursing facility or rehabilitation hospital.

Procedures. As part of a class assignment, participants were instructed to consume only thickened liquids for 24 hours. No further restrictions on food intake were made. Thickened liquids included milkshakes, smoothies, or any thin liquid mixed with a commercial thickening agent, such as Thick-1 ${ }^{\circledR}$. The students were provided with a supply of starch-based commercial thickening agents and provided with instructions concerning their use. A training session during which students practiced administration of thickener into a cup of water was included.

Twenty-four hours prior to the use of thickened liquids, the students were asked to keep a log of all foods/liquids consumed during this time frame. They were asked to keep the same log during the 24 hour period of thickened liquid intake. The intake logs were examined as an index of food/water consumed prior to and during the period of thickened liquid use.

At the end of the period of thickened liquid intake, each student was asked to write a brief narrative concerning their experience. The students were not guided to focus on any particular aspects of their experience in order to capture the most impartial data possible. While it is likely that most of the students had preconceived notions regarding thickened liquids given their prior exposure, it was not possible to control for the potential bias of these experiences within the current study.

Once the narratives were submitted, key terms were identified that seemed to define the participants' experiences. The key terms were chosen to reflect the most commonly reported experiences. The frequency of key term use was recorded by counting the number of times each identified term was used by a different participant; i.e., if one participant used the word "thirsty" several times in her narrative to describe a sensation experienced during the 24 hour period, then a single tally mark was recorded. For every subsequent participant who used the same term, an additional tally mark was recorded whether the participant wrote the term just once or multiple times. Related terms were grouped under primary headings for simplification. For example,

(C) The Internet Journal of Allied Health Sciences and Practice, 2015 
terms/phrases related to "thirsty" included "not quenched," "craved water," and "dry." The objective for identifying key terms and calculating frequency of use across participants was to determine the percent of participants who self-described similar experiences. The most commonly used terms derived from the narratives were grouped into three main categories: Physical Symptoms, Emotional Responses/Complaints, and Behavioral Changes.

Each author independently read and coded the student participants' narratives based on the key terms used to describe their experiences. Inter-rater reliability was analyzed by comparing the key term coding logs. Cohen's Kappa coefficient, a statistical measure of inter-rater agreement, was calculated for the three main categories of key terms: Physical Symptoms, Emotional Responses/Complaints, and Behavioral Changes. A Kappa statistic of 0.97 was attained for the Physical Symptoms category, as it was quite simple to interpret a participant's indication that he/she experienced thirst, tiredness, headaches, or UTI symptoms. A Kappa statistic of 0.66 was attained for the Emotional Responses/Complaints category. An explanation for lower agreement in this area would be that participants used many different, but similar, adjectives to describe their emotional responses. For example, words such as "unenjoyable," "terrible," and "disgusting" were used to describe the act of drinking thickened liquids. These examples would have been coded as "unpleasant." Although lower than the first category, reliability for the Emotional Responses/Complaints category was still substantial, according to Viera \& Garrett. ${ }^{18}$ A Kappa statistic of 0.77 was attained for the final category of Behavioral Changes. Overall, inter-rater reliability was good for the categorical data analyzed.

\section{RESULTS}

Within the narrative, $33 \%$ of the students wrote that they had thought the assignment would be fairly easy to complete. The remaining students either provided no information regarding their preconceived impressions (56\%) or thought that the assignment would be difficult (8\%). Two students (3\%) considered the assignment to be an "interesting" one to complete. All but one of the students reported a negative reaction to the assignment upon completion. Notably, all students reported a greater understanding of thickened liquid use upon completion; most stated that they would not recommend thickened liquids unless necessary.

Fifteen key terms were identified based on the participants most commonly reported experiences. The key terms fell into the three main categories previously mentioned (physical, emotional, and behavioral). As shown in Figure 1, the most common physical symptoms included feelings of thirst and fatigue, headache, and bladder changes. Additional symptoms reported by a small number of students ( $6 \%$ or less) included feeling ill, hoarse vocal quality, increased effort to swallow, and bad breath.

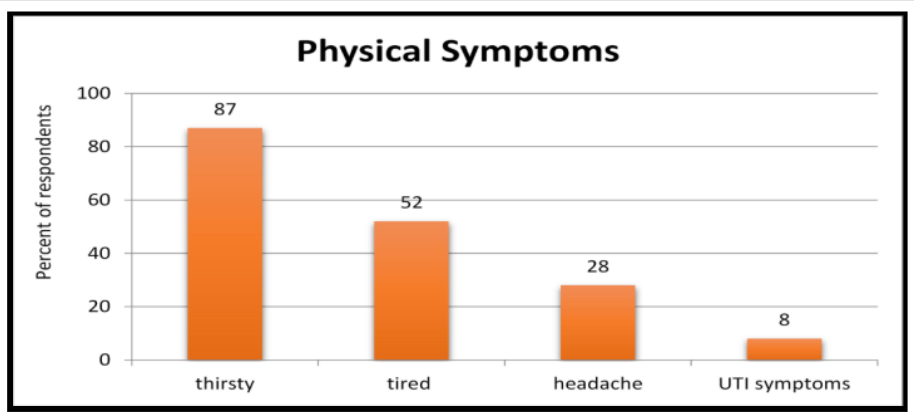

Figure 2 illustrates the most frequently occurring emotional responses or complaints associated with the use of thickened liquids. More than half of the students found it difficult to adhere to the thickened liquid protocol and reported a negative reaction to the experience. Additional reactions ( $6 \%$ or less) included that the use of thickened liquids was expensive, restrictive, and unrealistic.

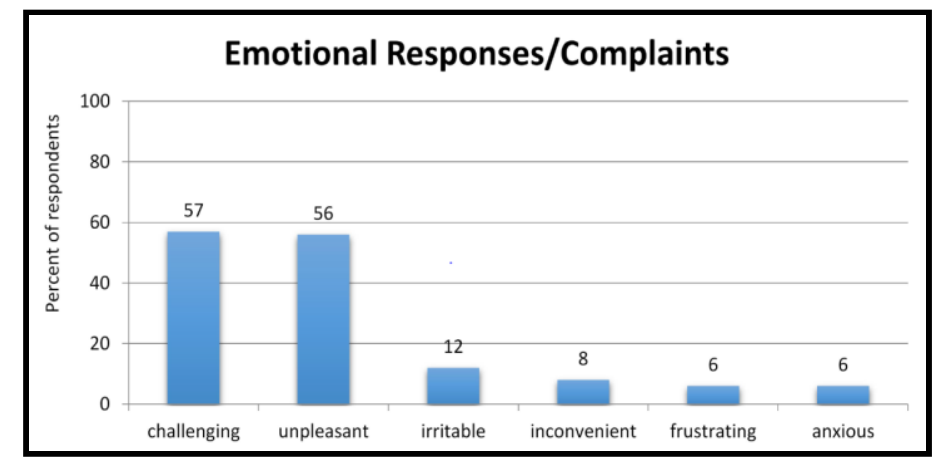

(C) The Internet Journal of Allied Health Sciences and Practice, 2015 
Finally, as can be seen in Figure 3, there were notable behavioral changes that occurred during the 24 hour period. Many students reported that they altered the manner or frequency of eating/drinking while following the thickened liquid protocol. Some students noted a reduced ability to concentrate or focus on their work during this time frame.

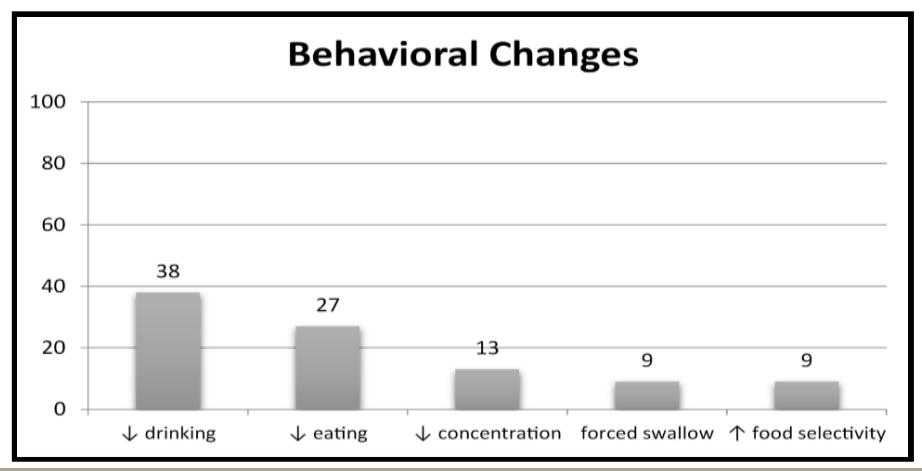

\section{DISCUSSION}

Most participants reacted negatively to the experience, stating that the 24 hour period of thickened liquid intake was difficult and unpleasant. These reactions were associated with a variety of factors, including the consistency of thickened liquids, a decrease in the amount of liquid and food consumed, feelings of dehydration associated with reduced water intake, and a constant desire to drink water. Several participants reported that they began to crave water once it became restricted, which is a common phenomenon when oral intake is restricted. ${ }^{19}$

An interesting reaction to the thickened liquid intake appeared to be a reduction in cognitive abilities, at least in the area of attentive abilities, as several students reported a reduced ability to concentrate. These symptoms were likely related to mild dehydration associated with reduced fluid intake. Indeed, pilot data has demonstrated decreased accuracy and speed in problem solving abilities in healthy young adults following a brief period of thickened liquid intake (Gorham-Rowan, unpublished data). As previously noted, mild dehydration can negatively impact cognitive function in healthy individuals and would likely impact cognition in patients with neurogenic impairments as well. ${ }^{14-16}$ Faraco et al found that dehydration disrupts cerebral blood flow in mice, thereby contributing to changes in cognitive function and potentially increasing the brain's vulnerability to cerebral ischemia. ${ }^{20}$ The impact of dietary changes upon rehabilitative potential and recovery in neurogenically impaired patients is not known and warrants further investigation.

Mild dehydration has been associated with reduced cerebral blood flow and associated ventricular enlargement as well as alterations in blood-brain permeability and neurotransmitter function. ${ }^{21-23}$ Kempton et al reported an increase in neural activity following dehydration in the dorsal fronto-parietal region that governs executive functions. ${ }^{23}$ However, this increase was not accompanied by a change in cognitive function. These data suggest that the participants exerted a higher level of neural function to maintain the pre-experimental levels. This pattern may be reflected in changes in perceived exertion in that participants may report increased fatigue and greater effort and concentration to complete a task. ${ }^{24} \mathrm{~A}$ similar phenomenon likely occurred in the present study for the students who reported difficulty concentrating and greater fatigue.

An additional outcome of the study was the change in perception of the use of thickened liquids for patients with dysphagia. Prior to initiation of thickened liquids for 24 hours, approximately one-third of the participants did not view the assignment as being difficult. However, upon conclusion of the designated time frame, over half of the students reported that the experience was challenging and/or unpleasant, frequently because their access to water was restricted. All students reported greater empathy for patients with dysphagia; most of them noted that they would recommend thickened liquids only after significant consideration of all options, for example, a free water protocol. ${ }^{25-27} \mathrm{~A}$ free water protocol includes unrestricted access to water for patients with dysphagia who may aspirate as long as certain conditions are met, such as proper oral hygiene and relative patient mobility. Free water protocols have been found to improve overall hydration status and quality of life in patients. ${ }^{26-27}$ For example, one student wrote, "I am glad that I was able to participate in the assignment because it opened my eyes to the daily struggles of patients on thickened liquid diets and how this could lead to a multitude of health problems." Another student commented, "Professionals involved in decision-making for patients should try to allow patients to be on the least restrictive diet according to their level of functioning." This shift in thinking suggests a deeper understanding of the ramifications of such a dietary change and can only serve to improve future clinical skills in these students and improve the quality of care for their patients. 


\section{CONCLUSION}

In this study, subjects (students) were required to consume only thickened liquids for a 24-hour period as an experiential learning activity. The use of this hands-on learning experience appeared to enhance students' understanding of the potential impact of thickened liquid recommendations on quality of life in patients with dysphagia.

\section{REFERENCES}

1. Garcia JM, Chambers E, Clark M, Helverson J, Matta Z. Quality of care issues for dysphagia: Modifications involving oral fluids. J Clin Nurs. 2010:19:1618-24. doi: 1111/j.1365-2702.2009.03009.x. [PMID 20384670]

2. Logemann JA. Evaluation and Treatment of Swallowing Disorders,2e. 1998. Austin, TX: Pro-Ed.

3. Sura L, Madhavan A, Carnaby G, Crary MA. Dysphagia in the elderly: Management and nutritional considerations. Clin Interv Aging. 2012:7:287-98. [PMID 22956864]

4. Goulding R, Bakheit AMO. Evaluation of the benefits of monitoring fluid thickness in the dietary management of dysphagic stroke patients. Clin Rehab. 2000:14:119-24. [PMID 10763787]

5. McGrail A, Kelchner LN. Adequate fluid intake in hospitalized stroke patients: Does viscosity matter? Rehab Nurs. 2012:37: 252-7. doi: 10.1002rnj.23. [PMID 22949278]

6. Vivanti AP, Campbell KL, Suter MS, Hannan-Jones MT, Hulcombe JA. Contribution of thickened drinks, food and enteral and parenteral fluids to fluid intake in hospitalised patients with dysphagia. J Hum Nutr Diet. 2009:22:148-55. doi: 10.1111/j.1365-277X.2009.00944.x. [PMID 19302120]

7. Whelan K. (2001). Inadequate fluid intakes in dysphagic acute stroke. Clin Nutr. 2001:20:423-8. [PMID 11534937]

8. Garcia JM, Chambers E, Molander M. Thickened liquids: Practice patterns of speech-language pathologists. Am J Sp Lang Path. 2005:14:4-13. [PMID 15962843]

9. Goulding R, Bakheit AMO. Evaluation of the benefits of monitoring fluid thickness in the dietary management of dysphagic stroke patients. Clin Rehab. 2000:14:119-24. [PMID 10763787]

10. Casa DJ, Stearns RL, Lopez RM, Ganio MS, McDermott BP, Walker Yeargin S, Yamamoto LM, Mazerolle SM, Roti, MW, Armstrong, LE, Maresh CM. Influence of hydration on physiological function and performance during trail running in the heat. J Athl Train. 2010:45:147-56. [PMID 20210618]

11. Grandjean AC, Grandjean NR. Dehydration and cognitive performance. J Am Coll Nutr. 2007:26(5 Suppl):549S-54S. [PMID 17921464]

12. Maughan RJ. Impact of mild dehydration on wellness and on exercise performance. Eur J Clin Nutr. 2003:57(Suppl 2):S1923. [PMID 14681709]

13. Szinnai G, Schachinger H, Arnaud MJ, Linder L, Keller U. Effect of water deprivation on cognitive-motor performance in healthy men and women. Am J Physiol Regul Integr Comp Physiol 2005:289:R275-80. [PMID 15845879]

14. Edmonds CJ, Crombie R, Ballieux H, Gardner MR, Dawkins L. (2013). Water consumption, not expectancies about water consumption, affects cognitive performance in adults. Appetite. 2013:60:148-53. [PMID 23104227]

15. Ganio MS, Armstrong LE, Casa DJ, McDermott BP, Lee EC, Yamamoto LM, Marzano S, Lopez RM, Jimenez L, Le Bellego L, Chevillotte E, Lieberman HR. Mild dehydration impairs cognitive performance and mood of men. Br J Nutr. 2011:106:1535-43. doi. 10.1017/S0007114511002005 [PMID 21736766]

16. Suhr JA, Patterson SM, Austin AW, Heffner KL. The relation of hydration status to declarative memory and working memory in older adults. J Nutr Health Aging. 2010:14:840-3. [PMID 211252021

17. Micheal J. Where's the evidence that active learning works? Adv Physiol Educ. 2006:30:159-67. doi:10.1152/advan.00053.2006. [PMID 17108243]

18. Viera AJ, Garrett JM. Understanding interobserver agreement: The kappa statistic. Fam Med. 2005:37:360-3. [PMID 15883903]

19. Hill AJ. The psychology of food craving. Proc Nutr Soc. 2007:66:277-85. [PMID 17466108]

20. Faraco G, Wijasa TS, Park L, Moore J, Anrather J, ladecola C. Water deprivation induces neurovascular and cognitive dysfunction through vasopressin-induced oxidative stress. J Cereb Blood Flow Metab. 2014:34:852-860. [PMID 24517977]

21. Costill DL, Fink WJ. Plasma volume changes following exercise and thermal dehydration. J Appl Physiol. 1974:37:521-5. [PMID 4415099]

22. Adan A. Cognitive performance and dehydration. J Am Coll Nutr. 2012:31:71-8. [PMID 22855911]

23. Kempton MJ, Ettinger U, Foster R, Williams SCR, Calvert GA, Hampshire A, Zelaya FO, O'Gorman RL, McMorris T, Owen AM. Dehydration affects brain structure and function in healthy adolescents. Human Brain Mapp. 2011:32:71-9. [PMID 20336685]

24. Szinnai G, Schachinger H, Arnaud MJ, Linder L, Keller U. Effect of water deprivation on cognitive-motor performance in healthy men and women. Am J Physiol Regul Integr Comp Physiol. 2005:289:R275-R280. [PMID 15845879]

(C) The Internet Journal of Allied Health Sciences and Practice, 2015 
25. Panther K. The Frazier free water protocol. Perspectives 2005:14:4-9.

26. Carlaw C, Finlayson H, Beggs K, Visser T, Marcoux C, Coney D, Steele CM. Outcomes of a pilot water protocol project in a rehabilitation setting. Dysphagia. 2012:27:297-306. [PMID 21928099]

27. Karagiannis M, Karagiannis TC. Oropharyngeal dysphagia, free water protocol and quality of life: An update from a prospective clinical trial. Hell J Nucl Med. 2014:17 Suppl1:26-9. [PMID 24392465] 